\title{
Custos da poluição atmosférica nas regiões metropolitanas brasileiras
}

\section{Costs of air pollution in Brazilian metropolitan regions}

Simone Georges El Khouri Miraglia ${ }^{1}$

Nelson Gouveia ${ }^{2}$

\footnotetext{
${ }^{1}$ Instituto de Ciências Ambientais, Químicas e Farmacêuticas, Universidade Federal de São Paulo. R. Prof. Artur Riedel 275, Jd. Eldorado. 09.972270 Diadema SP Brasil. miraglia@terra.com.br ${ }^{2}$ Departamento de Medicina Preventiva, Faculdade de Medicina, Universidade de São Paulo.
}

\begin{abstract}
This study evaluated the economic impact of health events associated with air pollution in Brazilian metropolitan regions. From the estimated mortality attributable to concentrations of particulate matter (PM) in 29 metropolitan areas, with a total of 20,050 deaths, the costs associated with mortality were calculated by means of the use of DALY (Disability Adjusted Life Years) methodology. The cost of premature deaths in Brazil resulted in \$ 1.7 billion annually. Translating losses in public health to economic values served to compare this data with the expense budget of the Ministry of Health and highlight priorities in decision-making of public policies that minimize the magnitude of these impacts.
\end{abstract}

Key words Air pollution, Mortality, Costs and cost analysis, Brazil
Resumo O presente estudo avaliou o impacto econômico de eventos de saúde associados com a poluição do ar em regiões metropolitanas brasileiras. A partir de estimativa da mortalidade atribuivel às concentrações de Material Particulado (MP) em 29 Regiões Metropolitanas, que totalizaram 20.050 óbitos, foram calculados os custos associados a essa mortalidade por meio da metodologia DALY (Disability Adjusted Life Years). O custo das mortes prematuras no Brasil resultou em US\$ 1,7 bilhão anualmente. A tradução de perdas em saúde pública para valores econômicos serviu para comparar com o orçamento de gastos do $\mathrm{Mi}$ nistério da Saúde e evidenciar as prioridades na tomada de decisão de políticas públicas que minimizem a magnitude desses impactos.

Palavras-chave Poluição do ar, Mortalidade, Custos e análise de custos, Brasil 


\section{Introdução}

Os efeitos à saúde relacionados à exposição à poluição atmosférica têm sido foco de estudos epidemiológicos em todo o mundo e, de modo geral, os resultados indicam associação entre essa exposição e a mortalidade e morbidade por diferentes causas, com destaque para as doenças cardiovasculares e respiratórias ${ }^{1}$.

As emissões atmosféricas que contaminam o ar podem ser provenientes de fontes fixas (indústria) e móveis (veículos automotores), impactando diretamente na qualidade do ar local, regional e global e afetando a saúde pública. Em 2012 a Organização Mundial da Saúde (OMS) estimou cerca de sete milhões de mortes no mundo decorrentes da poluição do ar a partir de fontes urbanas e rurais ${ }^{2-4}$.

No Brasil, até a década de 80 , a principal fonte de emissões de contaminantes do ar nas capitais brasileiras eram as indústrias. Já na década de 90 muitas empresas migraram para o interior e os veículos automotores passaram a ser a principal fonte de poluentes em conglomerados urbanos nos últimos anos ${ }^{5}$.

O controle da poluição por fontes móveis no Brasil iniciou-se com o Programa de Controle da Poluição do Ar por Veículos Automotores (PROCONVE) em 1986. A partir de 1995 foi estabelecido o controle da emissão de gases poluentes pelo escapamento de veículos. Atualmente, o programa está em sua sétima fase e, estabelece limites de emissões mais rígidos para veículos pesados, exigindo que tenham novas tecnologias ${ }^{6}$.

Atualmente, a frota veicular no Brasil totaliza 81 milhões de veículos e 18\% desta frota está concentrado nas regiões metropolitanas de São Paulo, Belo Horizonte, Salvador, Vitória, Curitiba, Campinas, Rio de Janeiro e Baixada Santista ${ }^{7}$.

Segundo a OMS, os altos níveis de poluição do ar são muitas vezes subproduto de políticas insustentáveis em setores como o de transportes. $\mathrm{Na}$ maioria desses casos, as estratégias mais saudáveis também são as mais econômicas em longo prazo, devido à redução de custos de cuidado com a saúde ${ }^{8}$.

Alguns exemplos de ganhos na saúde podem ser encontrados na bibliografia mundial, como a publicação de Roberts' ${ }^{9}$ que analisa o período entre 1997 e 2007 em que houve a aplicação de políticas governamentais cujas intervenções visavam à melhora da qualidade do ar e, como resultado, um potencial decréscimo da toxicidade do material particulado inalante $\left(\mathrm{MP}_{10}\right)$, um dos principais poluentes atmosféricos, ocasionando a diminuição de $10 \%$ na mortalidade anual por doenças cardiovasculares nas cidades de Brisbane e Sidney, Austrália.

Henschel et al. ${ }^{10}$ analisaram 28 estudos que demonstram que intervenções governamentais para a redução da poluição atmosférica possuem benefício direto na qualidade do ar e nos efeitos para a saúde, reduzindo a mortalidade e a morbidade cardiorrespiratória. Os efeitos observados excederam as previsões derivadas dos estudos de série temporal.

Os ganhos financeiros na saúde podem ser medidos contabilmente através da valoração econômica que tem se demonstrado uma ferramenta importante para avaliação de custo-benefício em saúde pública por auxiliar na tomada de decisões políticas voltadas à saúde ${ }^{11}$.

Os impactos e benefícios na saúde humana podem ser avaliados segundo duas categorias: morbidade e mortalidade. De acordo com a U.S Environmental Protection Agency, esses desfechos refletem em custos públicos e privados diretos, como a perda de produtividade (dias de trabalho perdidos), atividades restritas ou morte prematura e despesas médicas (tratamentos, exames e medicamentos) ${ }^{11}$.

No caso da valoração da saúde humana, pode-se comparar a "saúde" colocada como mercadoria com seu equivalente em termos de outra mercadoria, as unidades monetárias. A forma de avaliação pela utilidade de bens mercantis e pelo uso como principal meio de troca para diversos bens e serviços é a grande motivação do emprego de unidades monetárias como métrica para variações de risco e estado de saúde ${ }^{11}$.

O cálculo da perda de produtividade avaliada por meio da variação da produção, do rendimento e do consumo e a estimativa do valor monetário atribuído à redução do risco de morte são métodos que dizem respeito à valoração da mortalidade ${ }^{11,12}$.

Diálogos entre diversos interessados, sejam eles formuladores ou executores de políticas públicas, a população em geral ou os empreendedores e investidores dos diferentes setores econômicos do país ou região, podem resultar em esforços para implantação de políticas de mitigação da poluição atmosférica. Nesse cenário, associar valores econômicos aos custos com a saúde, como uma estimativa do custo total da doença ou à disponibilidade de se pagar por uma redução desses efeitos, é argumento eficiente para fomentar essa discussão.

Assim, o presente estudo objetiva avaliar o impacto econômico de eventos de saúde associa- 
dos com a poluição do ar em regiões metropolitanas brasileiras.

\section{Materiais e Métodos}

Neste estudo, a atribuição de valores monetários ao impacto na saúde da poluição atmosférica será feito através da metodologia DALY.

O método DALY (Disability Adjusted Life Years ou Anos de Vida Perdidos e Vividos com Incapacidades) é uma medida sumária de saúde expressa por um indicador padrão em unidade de tempo (anos), obtido pela soma de dois componentes: YLL - Years of Life Lost ou Anos de Vida Perdidos, em função de uma morte prematura (associada a um desfecho específico) correlacionada à expectativa de vida estimada e o YLD Years Lived with Disability ou Anos Vividos com Incapacidades, ou seja, o tempo vivido em condições de saúde não condizentes às ideais devido a desfechos como doenças, lesões ou a fatores de risco. A obtenção do componente YLL do DALY é apresentada na equação (1) ${ }^{13}$.

Como a estimativa dos efeitos à saúde causados pela exposição a poluentes atmosféricos foi determinada a partir do cálculo da mortalidade atribuída, a análise da valoração dos efeitos também está focada apenas nesse desfecho. Assim, serão estimados os DALY relacionados à mortalidade atribuível à exposição à poluição do ar e na sequência os DALY serão valorados.

\section{População de estudo}

Os custos monetários do impacto da poluição do ar na mortalidade foram contabilizados para dois cenários diferentes, a partir de estimativas calculadas no âmbito de um estudo sobre impactos à saúde decorrentes da poluição atmosférica em regiões metropolitanas, conduzido por Ponce de Leon (Instituto de Medicina Social da Universidade Estadual do Rio de Janeiro) $)^{14}$. Em resumo, no primeiro cenário foi utilizada a estimativa de óbitos anuais atribuíveis aos atuais níveis de contaminação atmosférica observados em oito Regiões Metropolitanas (RM) que dis- põe de dados de monitoramento da qualidade do ar (São Paulo, Belo Horizonte, Salvador, Vitória, Curitiba, Campinas, Rio de Janeiro e Baixada Santista).

O cálculo do número de mortes evitáveis foi realizado utilizando-se a abordagem sugerida pela Organização Mundial de Saúde (OMS) ${ }^{15}$. Esta abordagem baseia-se em frações atribuíveis que são calculadas usando equações já conhecidas para estimar o número de mortes evitáveis devido à poluição do ar.

O segundo cenário incluiu as demais RM brasileiras, segundo definição do IBGE, e que não dispunham de informações de qualidade do ar. Para estas foi construído um modelo de previsão dos níveis anuais de material particulado $\left(\mathrm{MP}_{10}\right)$ baseado no "Global Model of Ambient Particulates” (GMAPS), utilizado pelo Banco Mundial para apoiar os cálculos globais das avaliações de impacto da poluição do ar na saúde ${ }^{16}$. Resumidamente, a partir dos níveis de $\mathrm{MP}_{10}$ observados nas oito RM que dispunham dessa informação, e utilizando variáveis como, por exemplo, indicadores do perfil da frota de veículos e/ou mistura de combustíveis (\% diesel, \% gasolina, \% etanol etc.) consumidos pela frota local, consumo de energia por tipo de fonte per capita, renda per capita, perfil demográfico, variáveis meteorológicas, entre outras, foi possível estimar, por meio de modelo de regressão, os níveis médios de $\mathrm{MP}_{10}$ para 29 regiões metropolitanas brasileiras. Assumiu-se uma relação entre $\mathrm{MP}_{2,5} / \mathrm{MP}_{10}$ aproximadamente igual a 0,5 . Foi então estimado o impacto desses níveis de poluição na mortalidade geral.

De acordo com esse estudo, para as $8 \mathrm{RM}$ que dispõe de dados de monitoramento da qualidade do ar, foram estimados 15.164 óbitos anuais, atribuíveis aos atuais níveis de contaminação atmosférica. Para o cenário de dados previstos, a análise foi expandida e foram totalizadas 29 regiões metropolitanas, alcançando 20.050 óbitos anuais.

\section{Fonte de dados}

Para o cálculo do número de óbitos atribuíveis à poluição do ar foram utilizados os registros de mortalidade obtidos do Sistema de Informa-

$$
Y L L_{S}=\frac{K C e^{r a}}{(r+\beta)^{2}}\left[e^{-(r+\beta)(L+a)}[-(r+\beta)(L+a)-1]-e^{-(r+\beta) a}[-(r+\beta) a-1]\right]+\frac{1-K}{r}\left(1-e^{-r L}\right)
$$


ção de Mortalidade (SIM) para todos os municípios das RM incluídas no estudo. Os dados relativos às concentrações diárias de $\mathrm{MP}_{10}$ foram disponibilizados pela Companhia de Tecnologia de Saneamento Ambiental (CETESB) em São Paulo e pelo Instituto Estadual de Ambiente (INEA), no Rio de Janeiro. Para cidades pertencentes a Regiões Metropolitanas de outros estados, os dados ambientais foram obtidos junto aos órgãos ambientais locais.

Para a estimativa dos DALY, este estudo levou em conta a expectativa de vida da população, conforme publicado pelo Instituto Brasileiro de Geografia e Estatística (IBGE). O valor total de vidas perdidas prematuramente empregando a metodologia DALY pode ser expresso em termos econômicos utilizando o conceito de VOLY (value of life year), ou seja, o valor de um ano de vida perdido em resultado da mortalidade prematura comparada à expectativa de vida prevista. Essa abordagem consiste no resultado de estudos que estimam o valor de vida estatístico (VVE), isto é, a soma de preferências individuais resultante da atribuição a determinados riscos utilizando técnicas de valoração ambiental, como a disposição a pagar (DAP) ou a disposição a acei$\operatorname{tar}(\mathrm{DAA})^{17}$.

\section{Resultados}

\section{Mortalidade em Regiões Metropolitanas (RM) com monitoramento da qualidade do ar}

A fim de aplicar a metodologia DALY, os óbitos totais estimados foram divididos entre os gêneros masculino e feminino. Para essa estimativa, considerou-se que a mortalidade estava distribuída igualmente entre os gêneros, acometendo de forma proporcional homens e mulheres. Foi considerada a proporção etária no desfecho de mortalidade baseando-se na pirâmide populacional brasileira e, portanto, os óbitos totais nas regiões metropolitanas analisadas resultaram na divisão que consta na Tabela 1.

A Tabela 2 apresenta os resultados obtidos em termos de DALY e sua valoração.

Nesta etapa, estimaram-se os valores totais de prejuízos à saúde para a mortalidade por todas as causas, analisando-se todas as Regiões Metropolitanas conjuntamente (Belo Horizonte, Salvador, Vitória, Curitiba, São Paulo, Campinas, Rio de Janeiro e Baixada Santista). Considerou-se, portanto, as mortes atribuíveis ao $\mathrm{MP}_{25}$ apenas referentes à Tabela 1. Foi considerada a cotação do dólar de 02/02/2012.

Essas estimativas dos valores de custos de saúde foram valoradas e resultaram em US\$1.209.737.687,00 para o período de um ano.

\begin{tabular}{clc}
\hline $\begin{array}{l}\text { Tabela 1. Óbitos por todas as causas observados nas } \\
\text { Regiões Metropolitanas (RM) divididos por faixas } \\
\text { etárias e gênero. }\end{array}$ \\
\hline Gênero & Faixa etária & $\begin{array}{c}\text { Número de } \\
\text { óbitos ajustado }\end{array}$ \\
\hline Feminino & 30 a 59 anos & 410 \\
& 60 a 69 anos & 1.034 \\
& 70 a 79 anos & 2.189 \\
Masculino & mais de 80 anos & 3.949 \\
& 30 a 59 anos & 410 \\
& 60 a 69 anos & 1.034 \\
& 70 a 79 anos & 2.189 \\
& mais de 80 anos & 3.949 \\
& Total óbitos & $\mathbf{1 5 . 1 6 4}$
\end{tabular}

Tabela 2. DALYs atribuidos à exposição ao MP2.5 e seu valor (US\$) nas RM analisadas.

\begin{tabular}{llrr}
\hline \multicolumn{1}{c}{ Gênero } & \multicolumn{1}{c}{ Faixa etária } & DALY & \multicolumn{1}{c}{ Valor (dólar) } \\
\hline Feminino & 30 a 59 anos & 8777,780495 & $\$ 318.540 .409,84$ \\
& 60 a 69 anos & 17334,43873 & $\$ 88.073 .988,33$ \\
& 70 a 79 anos & 28209,32209 & $\$ 141.901 .695,23$ \\
Masculino & mais de 80 anos & 38563,33496 & $\$ 192.055 .422,78$ \\
& 30 a 59 anos & 8822,136321 & $\$ 45.274 .596,48$ \\
& 60 a 69 anos & 17416,07273 & $\$ 88.488 .759,89$ \\
& 70 a 79 anos & 28334,78301 & $\$ 142.532 .802,83$ \\
& mais de 80 anos & 38726,89863 & $\$ 192.870 .012,31$ \\
& Total & $\mathbf{1 8 6 1 8 4 , 7 6 7}$ & $\mathbf{\$ 1 . 2 0 9 . 7 3 7 . 6 8 7 , 6 9}$ \\
\hline
\end{tabular}




\section{Mortalidade em Regiões Metropolitanas (RM) com níveis estimados de poluição atmosférica}

A mesma análise foi conduzida para se estimar o ônus econômico considerando-se dados de mortalidade previstos para um ano em 29 regiões metropolitanas. Essas regiões são compostas pelas Regiões Metropolitanas (RM) de Agreste, Aracaju, Baixada Santista, Belém, Belo Horizonte, Campina Grande, Campinas, Carbonífera, Cariri, Curitiba, Florianópolis, Fortaleza, Goiânia, Grande São Luis, João Pessoa, Londrina, Macapá, Maceió, Maringá, Mato Grosso, Natal, Porto Alegre, Recife, Rio de Janeiro, Salvador, São Paulo, Sudeste Maranhense, Vale do Itajaí e Vitória (Tabela 3).

O total de óbitos para as RM com níveis estimados de poluição do ar foi, analogamente à situação das RM com níveis observados de poluição atmosférica, dividido por faixas etárias e gênero a fim de se proceder à análise e estimativa de DALY (Tabela 4).

Tabela 3. Óbitos por todas as causas previstos nas Regiões Metropolitanas expandidas (RM) divididos por faixas etárias e gênero.

\begin{tabular}{clc}
\hline Gênero & \multicolumn{1}{c}{ Faixa etária } & $\begin{array}{c}\text { Número de } \\
\text { óbitos ajustado }\end{array}$ \\
\hline Feminino & 30 a 59 anos & 542 \\
& 60 a 69 anos & 1.367 \\
& 70 a 79 anos & 2.895 \\
Masculino & mais de 80 anos & 5.221 \\
& 30 a 59 anos & 542 \\
& 60 a 69 anos & 1.367 \\
& 70 a 79 anos & 2.895 \\
& mais de 80 anos & 5.221 \\
& Total óbitos & $\mathbf{2 0 . 0 5 0}$ \\
\hline
\end{tabular}

Essas estimativas dos valores de custos de saúde foram valoradas e resultaram em US\$ 1.708.207.972,00 para o período de estudo em análise ( 1 ano) a partir da mortalidade prevista para as $29 \mathrm{RM}$ estudadas.

\section{Discussão}

A mortalidade total devido aos níveis de poluição atmosférica estimada para as Regiões Metropolitanas do estudo de 20.050 óbitos é um número alarmante, especialmente levando-se em consideração que se trata de uma estatística anual. A magnitude desse impacto pode também ser interpretada em termos econômicos a fim de proporcionar elementos para os tomadores de decisão avaliarem as alternativas de investimentos em políticas de saúde públicas que minimizem esse impacto.

Este estudo buscou avaliar o impacto econômico dos eventos de saúde associados com a poluição do ar nas regiões metropolitanas brasileiras e mostrou que seu ônus é muito alto. Entende-se que essa avaliação econômica represente um embasamento eficiente para a formulação de políticas de mitigação da poluição do ar, uma vez que monetizar os efeitos à saúde é uma forma de se criar indicadores para priorização e intervenção.

A análise de estimativas econômicas de perdas em saúde deve ser encarada como um ponto de partida para a análise do emprego dos recursos econômicos nas políticas públicas. $\mathrm{O}$ orçamento dos governos sempre é restrito com diversas alternativas e necessidades de investimentos. Ao se analisar o impacto econômico estimado decorrente dos níveis de poluição atmosférica na saúde pública das 29 Regiões Metropolitanas estudadas e comparando-se ao orçamento do Ministério da Saúde do Brasil de 2014, concluímos

Tabela 4. DALYs atribuídos à exposição ao $\mathrm{MP}_{2.5}$ e seu valor (US\$) nas RM expandidas analisadas.

\begin{tabular}{rlrr}
\hline \multicolumn{1}{c}{ Gênero } & \multicolumn{1}{c}{ Faixa etária } & \multicolumn{1}{c}{ DALY } & Valor (dólar) \\
\hline Feminino & 30 a 59 anos & 11603,79763 & $\$ 442.684 .879,60$ \\
& 60 a 69 anos & 22916,99975 & $\$ 123.638 .380,59$ \\
& 70 a 79 anos & 37307,44058 & $\$ 201.275 .541,64$ \\
\multirow{2}{*}{ Masculino } & mais de 80 anos & 50984,8498 & $\$ 275.065 .860,80$ \\
& 30 a 59 anos & 11662,43387 & $\$ 62.919 .424,57$ \\
& 60 a 69 anos & 23024,92401 & $\$ 124.220 .637,45$ \\
& 70 a 79 anos & 37473,36537 & $\$ 202.170 .714,34$ \\
& mais de 80 anos & 51201,09844 & $\$ 276.232 .533,25$ \\
& Total & $\mathbf{2 4 6 . 1 7 4 , 9 1}$ & $\mathbf{\$ 1 . 7 0 8 . 2 0 7 . 9 7 2 , 2 3}$ \\
\hline
\end{tabular}


que corresponde a $2 \%$ do orçado. Essa proporção é significativa, considerando que foi avaliado apenas para uma parcela do território nacional e que o impacto econômico estimado não contemplou gastos com internações, medicamentos e nem a redução da produtividade econômica das pessoas afetadas, sendo, portanto, um impacto subestimado.

Cabe lembrar ainda que os valores aqui apresentados podem ser ajustados em função da identificação de metodologias mais refinadas para qualquer das etapas envolvidas na avaliação de impacto da poluição do ar na saúde.

Políticas públicas empreendidas no sentido de melhorar a qualidade do ar, como, por exemplo, o uso de combustíveis mais limpos, a melhoria dos sistemas de transporte públicos, entre outras, trarão benefícios à população, que sofrerá menos efeitos em sua saúde, resultando em menores taxas de internações hospitalares e menores índices de mortalidade, além de poupar os recursos financeiros destinados à saúde. Adicionalmente, é importante haver um equilíbrio em termos de justiça ambiental de forma que exposições ambientais não acarretem padrões desiguais de saúde ${ }^{18}$. Uma possível forma de avaliar as injustiças ambientais em padrões de saúde é através de indicadores de eficiência de serviços de saúde no Brasil, analisando-os frente a parâmetros ambientais ${ }^{19}$.

Ressalta-se que a redução na emissão de poluentes atmosféricos, além do benefício imediato na saúde da população, traz também benefícios de longo prazo por meio da mitigação das mudanças climáticas que também estão associadas à poluição atmosférica ${ }^{20}$.

\section{Conclusão}

A valoração econômica dos danos ambientais da poluição atmosférica, traduzidos em termos de indicadores de saúde (DALY), revela um cenário favorável à implementação de tecnologias menos poluentes, políticas públicas, alteração na matriz energética, potencializando recursos para outros investimentos que devam priorizar a saúde pública da população exposta aos poluentes atmosféricos.

A análise conduzida nas principais regiões metropolitanas do país proporciona uma dimensão da atividade econômica do país e da magnitude de seus impactos em termos de poluição atmosférica, permitindo a condução de estudos adicionais orientativos para que se busque um desenvolvimento mais sustentável.

\section{Colaboradores}

SGEK Miraglia e N Gouveia participaram igualmente de todas as etapas de elaboração do artigo. 


\section{Referências}

1. Pope III CA, Dockery DW. Health Effects of Fine Particulate Air Pollution: Lines that Connect, Journal of the Air \& Waste Management Association 2006; 56(6):709-742.

2. Intergovernmental Panel On Climate Change (IPCC). Chapter 8: Human Health. IPCC. 2004 [acessado 2014 abr 04]; [cerca 15 p.]. Disponível em: http://www.ipcc. ch/pdf/assessment-report/ar4/wg2/ar4-wg2-chapter8. pdf

3. U.S. Environmental Protection Agency (EPA). Response to Comments on EPA's Designations and Classifications Of Areas for the Particulate Matter (PM2.5) National Ambient Air Quality Standards. EPA. 2013 [acessado 2013 out 23]; [cerca 33 p.]. Disponível em: http://www.epa.gov/airquality/particulatematter /2014/20140428fr.pdf

4. World Health Organization (WHO). 7 million premature deaths annually linked to air pollution. WHO. 2005 [acessado 2014 mar 14]. Disponível em: http:// www.who.int/mediacentre/news/releases/2014/air -pollution/en/

5. Toledo GIFM, Nardocci AC. Poluição veicular e saúde da população: uma revisão sobre o município de São Paulo (SP), Brasil. Rev. bras. epidemiol 2011; 14(3):445454.

6. Programa de Controle de Emissões Veiculares (Proconve). Controle de emissões. Proconve, Ibama. 2014 [acessado 2014 jan 7]. Disponível em: http://www.ibama. gov.br/areas-tematicas-qa/programa-proconve

7. Departamento Nacional De Trânsito (Denatran). Frota Nacional, 2013. 2013 [acessado 2014 mar 14]. Disponível em: http://www.denatran.gov.br/frota2013.htm

8. Organização Mundial da Saúde (OMS). 2014. World Health Organization. 7 million premature deaths annually linked to air pollution. WHO [on the Internet] 2005 [acessado 2014 mar]. Disponível em: http://www.who. int/mediacentre/news/releases/2014/air-pollution/en/

9. Roberts S. Have the short-term mortality effects of particulate matter air pollution changed in Australia over the period 1993-2007? Environ Pollut 2013; 182:9-14.

10. Henschel S, Atkinson A, Zeka A, Tertre AL, Analitis A, Katsouyanni K, Chanel O, Pascal M, Forsberg B, Medina S, Goodman PG. Air pollution interventions and their impact on public health. International Journal of Public Health 2012; 57(5):757-768.

11. U.S. Environmental Protection Agency (EPA). Valuing Mortality Risk Reductions for Environmental Policy: A White Paper. EPA. 2010 [acessado 2013 nov 11]. Disponível em: http://yosemite.epa.gov/sab\%5CSABPRODUCT.NSF/0/34D7008FAD7FA8AD8525750400712AEB/\$File/White+Paper+(Dec.+2010).pdf
12. Alberini A. Willingness to Pay for Mortality Risk Reductions: A Re-examination of the Literature. College Park: Final report to the US Environmental Protection Agency under Cooperative Agreement 015-29528; April, 2004.

13. Murray CJL, Lopez AD. The Global Burden of Disease: a comprehensive assessment of mortality and disability from diseases, injuries, and risk factors in 1990 and projected to 2020. Boston: Harvard School of Public Health; 1996.

14. Ponce de Leon A, Junger W, Gouveia N. Avaliação do impacto dos poluentes atmosféricos decorrentes das emissões veiculares do óleo diesel e gasolina na saúde, incluindo sua valoração [relatório de pesquisa]. Rio de Janeiro: Petrobras; 2012.

15. Baker D, Nieuwenhuijsen MJ. Environmental Epidemiology: Study methods and application. New York: Oxford; 2008.

16. Cohen AJ1, Ross Anderson H, Ostro B, Pandey KD, Krzyzanowski M, Künzli N, Gutschmidt K, Pope A, Romieu I, Samet JM, Smith K. The global burden of disease due to outdoor air pollution.J Toxicol Environ Health A 2005; 68(13-14):1301-1307.

17. Pearce D. Valuing Statistical Lives. Planejamento e Políticas Públicas 1998; 18(1):69-121.

18. Porto MFS, Milanez B. Eixos de desenvolvimento econômico e geração de conflitos socioambientais no Brasil: desafios para a sustentabilidade e a justiça ambiental. Cien Saude Colet 2009; 14(6):1983-1994.

19. Viacava F, Almeida C, Caetano R, Fausto M, Macinko J, Martins M, José Noronha JC, Novaes HMD, Oliveira ES, Porto SM, Silva LMV, Szwarcwald CL. Uma metodologia de avaliação do desempenho do sistema de saúde brasileiro. Cien Saude Colet 2004; 9(3):711-724.

20. Cifuentes L, Borja-Arbuto V, Gouveia N, Thurston G, Davis DL. Hidden health benefits of greenhouse gas mitigation. Science 2001; 293(5533):1257-1259.

Artigo apresentado em 05/07/2014

Aprovado em 11/07/2014

Versão final apresentada em 12/07/2014 